\title{
Kernos
}

Revue internationale et pluridisciplinaire de religion grecque antique

5 | 1992

Varia

\section{Épiménide sans paradoxe}

\section{Marie-Christine Leclerc}

Édition électronique
URL : http://journals.openedition.org/kernos/1063

DOI : 10.4000/kernos. 1063

ISSN : 2034-7871

\section{Éditeur}

Centre international d'étude de la religion grecque antique

\section{Édition imprimée}

Date de publication : 1 janvier 1992

ISSN : 0776-3824

\section{Référence électronique}

Marie-Christine Leclerc, «Épiménide sans paradoxe », Kernos [En ligne], 5 | 1992, mis en ligne le 19 avril 2011, consulté le 01 mai 2019. URL : http://journals.openedition.org/kernos/1063 ; DOI : 10.4000/kernos. 1063 


\section{ÉPIMÉNIIDE SANS PARADOXE}

La figure d'Épiménide le Crétois appartient à une catégorie connue et familière, celle du "mage inspiré", dont il est "le type même» ${ }^{1}$, doté de tous les attributs du prophète, purificateur et sage dont témoignent les documents recueillis par Diels ${ }^{2}$. Les tentatives pour reconstituer l'historicité de celui qui fut, selon Plutarque et Diogène Laërce, l'un des Sept Sages $^{3}$, n'aboutissent toutefois pas à des résultats véritablement satisfaisants.

Fut-il contemporain de Solon, comme, à la suite d'Aristote et de Plutarque ${ }^{4}$, le pensent la plupart des commentateurs ${ }^{5}$ ? Vint-il à Athènes dix ans avant les guerres Médiques, comme l'affirme Platon ${ }^{6}$, ou bien les Lois se font-elles l'écho indirect, comme le suggère $\mathrm{H}$. Demoulin, de tentatives politiques intéressées à la récupération de cette grande figure ${ }^{7}$ ? P. Faure avance les dates de $560-500^{8}$, pendant que L. Moulinier suppose qu'il y eut trois Épiménides, dont seul le dernier, vers 500 , serait historique ${ }^{9}$.

Épiménide était-il son nom ou celui d'un héros athénien qu'il aurait adopté en venant purifier la ville ${ }^{10}$ ? La nymphe Blastè, sa mère selon Plutarque et la Souda ${ }^{11}$, était considérée jadis par Usener comme la

J.P. Vernant, Les origines de la pensée grecque, Paris, 1962, p. 62.

2 L'édition utilisée ici est celle des Fragmente der Vorsokratiker de H. Diels et W. Kranz de 1951.

3 PluT., Banquet des Sept Sages; de même DIOG. LAËRCE, I, 109-115.

4 ARISTOTE, Constitution d'Athènes, I; PLUT., Solon, XII, 7, 12.

5 Voir H. Demoulin, Epiménide de Crète, Bruxelles, 1901 (Bibl. de la faculté de philosophie et lettres de l'Univ. de Liège, 12), p. 38-40; VERNANT, op. cit., p. 6872.

6 Platon, Lois, 642d; voir aussi KERN, art. Epimenides 2), in RE, VI (1909), c. 174.

7 DEMOULIN, op. cit., p. 43-50, 105.

8 P. FAURE, Fonctions des cavernes crétoises, Paris, 1964, p. 130.

9 L. Moulinier, Le pur et l'impur dans la pensée grecque d'Homère à Aristote, Paris, 1952, p. 51-57; voir aussi DeMoulin, op. cit., p. 134-135.

10 KeRN, ibid.

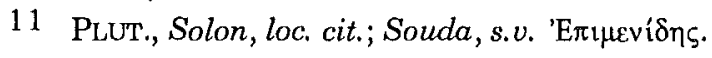


génitrice de son homonyme athénien ${ }^{12}$. Mais, selon T. Poljakov, le nom de Blastè appartiendrait au fond sémitique de l'ancien crétois ${ }^{13}$.

On pense tenir un point d'ancrage sûr dans l'origine crétoise du personnage, même si on ne sait trop s'il est de Cnossos ou de Phaïstos ${ }^{14}$. Les appellations d'Aiakos ou de Nouveau Courète qui lui sont attribuées le rapprochent du Zeus honoré sur l'île, note W. Burkert pour qui, comme pour R.F. Willetts, Épiménide est bien enraciné dans la tradition cultuelle des cavernes ${ }^{15}$. Demoulin cite Le crétois, drame perdu d'Euripide, dans lequel l'abstinence en matière alimentaire était présentée comme un trait spécifiquement crétois ${ }^{16}$. Cependant le végétarisme du mage et ses contacts divins pendant son sommeil dans la grotte pourraient aussi bien venir du nord, d'après E.R. Dodds, qui reconnaît des éléments thraces et l'influence du chamanisme sur l'un comme sur l'autre de ces caractères essentiels du personnage ${ }^{17}$. Ainsi, les certitudes sur son origine crétoise sont ébranlées par l'analyse précise et bien informée du savant anglais. Par ailleurs, les points communs entre Épiménide et les Pythagoriciens, qui ne sont pas crétois, sautent aux yeux ${ }^{18}$.

Il n'est dès lors plus si sûr qu'à travers les actes qu'on lui prête, Épiménide représente l'introduction en Grèce continentale des cultes crétois, que l'on verrait s'installer à Athènes lors de la purification ${ }^{19}$, et dont les relations avec Delphes seraient attestées dès l'Hymne homérique à Apollon ${ }^{20}$. Demoulin reconnaît dans la figure du sage celle, plus générale, d'un réformateur religieux. J.P. Vernant associe à ce rôle celui du réformateur politique soucieux de donner aux citoyens, en

12 Voir Demoulin, op. cit., p. 93.

13 T. PolJakov, The Nymph Baltè, mother of Epimenides, in $R h M, 130$ (1987), p. 410-412.

14 Diog. LAËrCE, I, 109, et PAUS., I, 14, 4 indiquent Cnossos; PlUT., Solon, loc. cit. et Strabon, X, 4, 14 penchent pour Phaistos. Voir DEMoulin, op. cit., p. 91 et n. 3, 4.

15 W. Burkert, Weisheit und Wissenschaft, Studien zu Pythagoras, Philolaos und Platon, Nuremberg, 1962, p. 127; R.F. WILLETTs, Cretan cults and festivals, London, 1962, p. 216, 242.

16 Demoulin, op. cit., p. 66.

17 E.R. DoDDs, The Greeks and the Irrational, Berkeley, 1959, trad. française Les Grecs et l'irrationnel, Paris, 1965, p. 141-146.

18 Voir en particulier M. DetienNe, Les jardins d'Adonis, Paris, 1972, p. 89-92.

19 WILLETTS, op. cit., p. 311-312.

20 Hymne hom. à Apollon, 392-544; WILLETTS, p. 263. 
ces périodes de crise des VIIe-VIe siècles, le sentiment de la collectivité qu'ils constituent ${ }^{21}$.

Une influence d'Épiménide et de la Crète sur la philosophie est également supposée par Faure, qui trouve la trace de l'Ida et des conditions de l'inspiration épiménidienne dans la configuration de la caverne platonicienne comme dans le caractère ésotérique que les philosophes prêtaient au savoir ${ }^{22}$. Cependant la Théogonie attribuée à Épiménide semble avoir eu recours à la fois à des traits hésiodiques, à des éléments orphiques et à des principes nouveaux comme l'air d'Anaximène. Cet ouvrage s'inscrirait dans l'effort mené à la fin du VIe siècle pour concilier les anciennes manières de penser et la philosophie naissante 23 . Mais Diels, qui les rassemble, considère tous les fragments attribués à Épiménide comme des faux orphiques, Théogonie incluse $e^{24}$.

Ces incertitudes et distorsions sont le rézultat d'une problématique positiviste, qui se propose d'établir la carte d'identité d'un homme supposé a priori personnellement identifiable, ou de ranger ses actes dans une catégorie de faits historiques matériellement isolables. En réalité, la cohérence du personnage d'Épiménide défie ce genre d'analyse, car il est impossible d'isoler, dans les légendes qui se sont constituées autour de lui dès le IVe siècle av. J.-C. ${ }^{25}$, ce qui est historiquement acceptable à nos yeux de ce qui relève du merveilleux. Ce que O. Kern et Demoulin appelaient «le roman d'Épiménide», ces «fictions qui se sont forgées autour» du Crétois ${ }^{26}$, constituent non des scories à éliminer, comme espérait le faire Demoulin, mais la matière même dont le sage est pétri. Selon les termes de Dodds, il ne reste rien d'Épiménide que la légende, mais une légende significative ${ }^{27}$. Demoulin l'avait bien vu, malgré tout, en relevant l'intérêt de ces fables pour l'étude de la pensée grecque et de son évolution ${ }^{28}$.

21 Demoulin, op. cit., p. 110-112; VERNANT, op. cit., p. 68-72. Voir aussi la remarque d'ARISTOTE, Politique, A 2.1252 b 13.

22 FAURE, op. cit., p. 249-250.

23 Demoulin, op. cit., p. 121-126; voir aussi FAURE, op. cit., p. 87, 96, 130.

24 Voir la documentation de Demoulin, op. cit., p. 66.

25 Demoulin, p. 51-52, 80; Pausanias, loc. cit., atteste que les légendes d'Épiménide étaient encore bien vivantes au IIe siècle de notre ère.

26 KERN, R.E, loc. cit.; DEMOULIN, op. cit., p. 136-137.

27 DoDDs, op. cit., p. 141-146.

28 DEMOULIN, op. cit., p. 136-137. 
Si j'osais, je dirais volontiers que les inscriptions apparues post mortem sur la peau d'Épiménide ne sont pas, comme croit Demoulin ${ }^{29}$, l'image d'un manuscrit consignant des prédictions, genre dont, aux dires d'Aristote, il n'était pas spécialiste ${ }^{30}$, mais une métaphore invitant à lire son corps comme un parchemin où se seraient inscrites les leçons que la tradition a retenues de lui, quelque chose comme un «miroir d'encre». L'analyse qui va suivre tente d'en mettre en lumière l'une des facettes.

Parmi les citations, peu nombreuses, de textes attribués à Épiménide, le vers aux Crétois menteurs est incontestablement le plus célèbre et le plus malmené. En totalité ou partiellement, on utilisa ce passage à des fins intéressées.

C'est d'abord le cas en matière religieuse. Ainsi Callimaque en cite

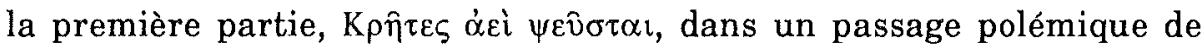
son Hymne à Zeus dans lequel il défend à la fois l'origine arcadienne et l'immortalité du dieu contre un Zeus Crétois dont on montrait la tombe $^{31}$. Le poète ne rapporte pas la formule à Épiménide, et É. Cahen estime que "ce début d'hexamètre» était "devenu proverbe»32. À l'inverse saint Paul attribue la totalité du vers à son auteur dans l'Épître à Tite, où il écrit à propos des Crétois : "L'un d'entre eux, leur

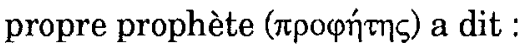



(Crétois toujours menteurs, méchantes bêtes, ventres paresseux). $\mathrm{Ce}$ témoignage est vrai» ${ }^{33}$. Plus tard, Clément d'Alexandrie citera l'épître et identifiera ce "prophète» comme étant Épiménide ${ }^{34}$. Il s'agissait pour l'apôtre de mettre en garde son correspondant contre les propagateurs de fausses croyances qui, selon lui, pullulaient dans l'île ${ }^{35}$. Le prosélytisme de saint Paul trouvait évidemment son compte dans l'admonestation d'Épiménide. Il n'en reste pas moins que celle-ci est présentée

29 Demoulin, op. cit., p. 69. Cette bizarrerie est rapportée par la Souda, loc. cit.

30 ARISTOTE, Rhétorique, $\Gamma$ 17. 1418a 21, affirme que la connaissance du sage portait sur le passé.

31 CALlim., Hymne à Zeus, 8-9.

32 Callimaque, Epigrammes, Hymnes, éd. É. Cahen, Paris, Belles Lettres, 1953, p. 210, n. 1.

33 SAINT PAUL, Epître à Tite, I, 12.

34 Clem. AleX., Stromates, I, 59.

35 SAINT PAUL, op. cit., I, 10, 14. 
comme un argument et son auteur comme un "prophète», le seul "païen" à se voir honoré de ce titre dans le Nouveau Testament ${ }^{36}$.

Si l'on en croit l'analyse que fait $P$. Courcelle d'une notice d'Isho'dad de Merv, où le vers d'Épiménide est cité en compagnie de ceux de Callimaque et d'un autre d'Aratos, certains débats théologiques continuèrent à avoir recours au $(x)$ Crétois. On a supposé qu'entrait dans ces discussions un passage plus étendu d'Épiménide que tentèrent de restituer R. Harris et A.B. Cook ${ }^{37}$. J'avoue mon peu d'enthousiasme tant pour ces restitutions que pour leur attribution au sage. Rien ne prouve en effet qu'Isho'dad, qui a quelque quinze siècles de moins qu'Épiménide et douze de moins que Callimaque et Aratos, n'a pas rapproché des textes qui, à ses yeux ou à ceux d'un prédécesseur dont il s'inspire, présentaient des points communs; rien ne permet d'affirmer que ces quatre vers formaient un tout dès Épiménide. Le plus intéressant, me semble-t-il, est qu'au IXème siècle l'évêque nestorien de Hadatha soit encore en mesure, malgré des confusions, de citer le texte exactement. Son utilisation par les Stoïciens pourrait avoir assuré la transmission du sage Crétois aux théologiens chrétiens ${ }^{38}$.

Les Stoïciens passent aussi pour avoir planché sur la première partie du vers, utilisée comme majeure d'un syllogisme aporétique. Ils seraient en cela les ancêtres des logiciens modernes, qui attribuent à Épiménide la paternité du paradoxe du menteur, redécouvert, semble-til, par B. Russel dans ses Principles of Mathematics ${ }^{39}$. Cette attribution pose problème. Car s'il est vrai que la majeure du syllogisme s'inspire sans doute du vers d'Épiménide, on ne lit en revanche nulle part que le sage Crétois se serait impliqué personnellement dans l'affaire en s'affirmant menteur, fût-ce par "plaisanterie» 40 . Or, à défaut d'une formulation de la mineure à la première personne, il n'y a plus aporie, mais syllogisme catégorique banal. C'est pourquoi $\mathrm{A}$. Koyré considère que cette formulation est imparfaitement paradoxale, et lui préfère la forme simplifiée adoptée par les logiciens médiévaux : "si quis dicat se mentire, an mentiat seu verum dicat» ${ }^{41}$. Or ceux-ci ne sont pas éloignés

36 Glossaire de la traduction œcuménique, s.v. prophète.

37 P. Courcelle, Un vers d'Epiménide dans le Discours de l'Aréopage, in REG, 76 (1963), p. 404-413, notamment p. 406-407.

38 CoURCELLE, p. 413.

39 Les Principia datent de 1910-1913. Voir l'analyse d'A. KoYRÉ, Epiménide le Menteur, Paris, 1947, notamment ici p. 5.

40 Koyré estime que l'histoire du menteur était une "plaisanterie grecque», p. 24.

41 KOYRÉ, p. 8-9. 
de la formulation cicéronienne du menteur, qui utilise le tutoiement ${ }^{42}$ : dans les deux cas, le locuteur est impliqué dans l'assertion. Quant à la forme “je mens", variante ramassée de la précédente, elle est considérée par Koyré comme essentiellement différente du syllogisme aux Crétois ${ }^{43}$, et à vrai dire elle est la seule aporétique. Mais cette forme parfaite du paradoxe n'est pas d'Épiménide. Selon P. de Rouilhan, c'est l'œuvre d'Eubulide de Milet ${ }^{44}$, à qui Diogène Laërce attribue, en effet, la paternité du paradoxe du menteur ${ }^{45}$. Cicéron en rapporte les difficultés à Chrysippe 46 . Or tant Diogène, l'une des principales sources à son sujet, que Cicéron, qui le cite dans le De divinatione 47 , connaissent Épiménide et ses talents. Il serait stupéfiant qu'ils n'aient pas mentionné le nom du sage à l'occasion du paradoxe si la tradition le lui avait attribué. En réalité il semble impossible de ne pas conclure que les logiciens, principalement les logiciens modernes, utilisent le nom d'Épiménide comme un identificateur (ainsi fait de Rouilhan luimême, bien qu'il sache à quoi s'en tenir), sans se soucier de la valeur historique de cette attribution ${ }^{48}$. Sans doute est-ce pourquoi hellénistes et logiciens s'ignorent superbement. Il n'est pourtant pas sans intérêt que ces derniers se réfêrent au vieux sage, dont l'autorité donne quelque poids à leurs activités ludiques, comme elle a autrefois servi à étayer des argumentations théologiques. C'est là sans doute une récupération intéressée, mais sans doute aussi une contribution à la légende d'Épiménide, contribution aussi tardive qu'imprévisible, le sage Crétois étant le contraire d'un menteur.

Quoi qu'en aient pensé autrefois certains critiques, supposant que le vers était l'œuvre d'un autre ${ }^{49}$, l'objurgation adressée aux Crétois fait bel et bien partie intégrante de la figure d'Épiménide, qui seule nous

42 Cic., Premiers Académiques, II, 30.

43 KoYRÉ, p. 15.

44 P. de Roullhan, Paradoxes légers, paradoxes lourds, in Pour la Science, 156 (octobre 1990), p. 98-103.

45 DIOG. LAËRCE, II, 108-112.

46 Crc, loc, cit.

47 Diog. L., I, 109-115; CIC., De divinatione, I, 18, 34.

48 On peut lire aussi l'article de C. IMBERT, À propos du menteur crétois, cet agent double, in Traverses, 47 (novembre 1989), p. 99-110, dont l'auteur est moins prudent que de Rouilhan.

49 Outre l'attribution aux orphiques, déjà citée, il faut nommer Musée, à qui Kern attribue le vers ( $R E$, loc. cit., c. 176), et la Pythie, qui en serait l'auteur selon J.G.M. LEE, Epimenides in the Epistle to Titus $(I, 12)$, in Novum Testamentum, $22(1980)$, p. 96. 
intéresse ici. On s'accorde à reconnaître l'influence d'Hésiode, dont le sage aurait été, dit-on parfois, le disciple ${ }^{50}$, sur la formulation de ce passage comme sur la pensée qui y est exprimée. Le vers du «maître» est le suivant :



Rappelons pour mémoire celui du «disciple»:

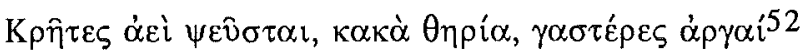

Même juxtaposition de trois groupes nominaux, scansion identique. Les bergers dans un cas, les Crétois dans l'autre, premiers mots du vers, donnent lieu à une appréciation visiblemement péjorative.

Épiménide reprend à Hésiode deux termes, en position identique : $\kappa \alpha \kappa \alpha \dot{\alpha}$, "mauvais, méchant", et $\gamma \alpha \sigma \tau \varepsilon \dot{\varepsilon} \rho \varepsilon$, "ventre, estomac". Chez

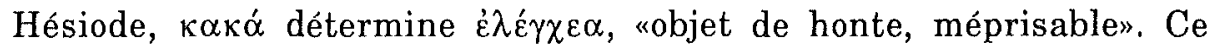
dernier terme trouve son explication dans les deux autres groupes constituant le vers. Les bergers sont $\alpha$ $\gamma \rho \alpha v \lambda_{o l}$, «demeurant dans les pâtures», dans l'ỏ $\gamma$ ós, zone située à la limite du sauvage, en compagnie de leurs bêtes. Ils sont aussi «uniquement des ventres». C'est leur bestialité qui est ici soulignée et qui d'ailleurs s'oppose au double savoir des Muses qui lui fait immédiatement suite ${ }^{53}$.

Ce ne sont toutefois pas les seuls bergers, mais l'ensemble des hommes actuels, après la "séparation des hommes et des dieux» ${ }^{54}$, qui sont des "ventres" chez Hésiode. Dans l'épisode prométhéen de la Théogonie, qui conte cette séparation, la part du bœuf réservée aux hommes (les parties consommables de la bête recouvertes de son "estomac") symbolise leur statut de mangeurs. Ils doivent également avoir recours au "ventre" de la femme, invention récente et intégrée dans le mythe de Prométhée, pour avoir une descendance. Ainsi la $\gamma \alpha \sigma \tau$ in résume-t-elle ce qu'a de bestial la condition humaine; le terme

50 PluT., Banquet des Sept Sages, 158b. Sur le type d'influence notée par Platon, Lois, $677 \mathrm{~d}$-e, voir ci-dessous.

51 Hes., Théog., 26.

52 Fr. Diels-kranz 1.

53 Hes., Théog., 27-28. Sur le sens d'áypós, voir P. Chantraine, Dictionnaire étymologique de la langue grecque, Paris, 1968 , s.v., $\dot{\alpha} \gamma p o ́ s$.

54 C'est le sens de "se séparer" qu'il faut attribuer au verbe $\kappa \rho i ́ v \omega$ dans


prométhéen de la Théogonie. Voir P. ChanTraine, Dictionnaire, s.v. кpív $\omega$, et M. Hofinger, Lexicon hesiodeum, Leiden, 1975-1978, s. v. кpívw. 
est d'ailleurs réservé aux hommes et aux animaux, chez Hésiode, qui emploie pour le ventre des dieux le terme $\eta_{\eta} \delta \delta^{5}{ }^{55}$.

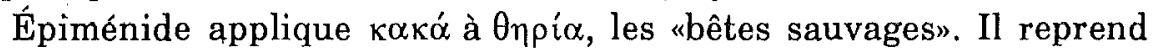
ainsi, en les faisant passer du premier groupe au second, les connotations de sauvagerie et de bestialité que comportait l'adjectif hésiodique



Hésiode emploie deux fois le mot $\theta$ íp, dont dérive Onpíov. Dans un cas, il s'agit du tableau de l'hiver, le seul dans les Travaux et les Jours, à présenter la nature non cultivée. Forêt, chênes et sapins, montagne, bêtes frissonnantes, hôtes des bois, ainsi que le fameux «sans os» dans sa maison "sans feu", s'opposent aux femmes dans leur confortable demeure ${ }^{56}$. C'est une autre composante de la bestialité et de la sauvagerie qui domine dans le second passage : aux bêtes, Zeus n'a pas donné la


phagie n'est d'ailleurs pas sans rappeler l'autophagie du "sans os" qui «ronge son pied» 58 .

Ce champ de représentations hésiodiques oppose évidemment les bêtes sauvages aux hommes civilisés, qui non seulement ont des maisons et des manteaux pour se protéger du froid ${ }^{59}$, mais aussi des normes alimentaires. Les travaux de Vernant ont montré la valeur étiologique de l'instauration du sacrifice et de l'agriculture dans l'épisode prométhéen ${ }^{60}$. L'alimentation céréalière et carnée constitue une réglementation des appétits qui est propre aux hommes. Ceux-ci sont des «ventres», mais il ne leur est pas permis de consommer n'importe quoi; c'est en cela qu'ils sont civilisés.

En employant $\theta \eta p i \alpha$, Épiménide va donc plus loin qu'Hésiode en ce qu'il semble retirer aux humains (ou aux seuls Crétois) leur statut de civilisés. Ce choix sémantique entre dans un ensemble qui rappelle également l'arrière-plan hésiodique. Les «ventres» d'Épiménide sont dits $\dot{\alpha} \rho \gamma \alpha \hat{i}$, «inactifs, paresseux». Hésiode connaît ce terme sous la forme $\dot{\alpha} \varepsilon$ yóc. Il l'applique deux fois à des bêtes, les frelons, qui, «se

55 Théog., 460, 487, 890, 899.

56 Trav., 504-563. Les «betes sauvages» figurent au vers 512.

57 Trav., 276-278.

58 Trav., 524.

59 Trav., 520, 536-546.

60 J.P. VERNANT, Le mythe prométhéen chez Hésiode, in Mythe et société en Grèce ancienne, Paris, 1974, p. 177-194; $\grave{A}$ la table des hommes, in M. Detienne et J.P. Vernant, La cuisine du sacrifice en pays grec, Paris, 1979, p. 37-132. 
refusant au travail... dévore(nt) le labeur des abeilles» ${ }^{61}$, eux qui, dans la Théogonie, "engrangent dans leur ventre le fruit des peines d'autrui»62. Dans les deux cas, il s'agit moins des frelons eux-mêmes que des humains auxquels ils sont comparés, la femme dans le second cas, dite aussi "à l'affût de la table» 63 , l'ókpyós, le paresseux, dans le premier. Cet inactif s'incarne dans la figure de Persès et de ses semblables, qui réussissent parfois à obtenir leur pitance en mendiant auprès de leurs proches, mais qui sont surtout menacés par la faim "faute d'avoir travaillé" pour emplir leur grange ${ }^{64}$. Ils ne se conforment pas à la loi que Zeus a imposée aux mortels en choisissant la part inconsommable du bœuf sacrifié par Prométhée : obligés de manger pour vivre, les hommes ne peuvent se contenter de "travailler un seul

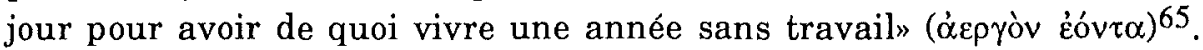
Le labeur est leur loi parce que le "ventre» est l'organe emblématique de leur condition : ils sont "uniquement des ventres» à remplir. Mais, pour Hésiode, cette obligation évite aux hommes de trouver leur pitance à la manière des bêtes sauvages, et c'est par leur travail qu'ils se distin-


qu'ils se distinguent des dieux immortels. Les hommes d'Hésiode se situent "entre bêtes et dieux", comme dit Vernant, et la loi du travail comporte l'ambiguité fondamentale de rendre les hommes chers aux dieux en leur imposant d'assumer leur condition de "ventres» 66.

Chez Hésiode, ce champ de représentations, pour être cohérent, n'en


et oîo, Épiménide le concentre en quatre mots. Il fait des Crétois des êtres qui penchent inexorablement vers la bestialité et la sauvagerie, enclins à toutes les mauvaises tendances contre lesquelles Hésiode met en garde son auditoire.

L'expression qui ouvre le vers accuse enfin les Crétois d'être

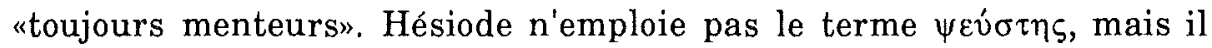
connaît bien le mensonge, qui est un trait du genre humain. Ce n'est pas que les dieux ne puissent mentir. Mais si cela leur arrive, ils sont

\footnotetext{
61 Trav., 305-306.

62 Théog., 599.

63 Trav., 704.

64 Trav., 302, 498.

65 Trav. 43-44.

66 Sur cette ambiguitté fondamentale, voir J.P. VERNANT, Entre bêtes et dieux, introduction aux Jardins d'Adonis de M. DeTIEnNe, et Raisons du mythe, in Mythe et société en Grèce ancienne., p. 195-250.
} 
sévèrement punis, et réduits pour un an à l'état de cadavres, "gisant sans souffle et sans voix", privés temporairement des nourritures divines; leur mensonge est d'ailleurs donné comme éventuel dans le groupe ós $\tau_{\imath \varsigma} \psi \varepsilon v ́ \delta \eta \tau \alpha \imath{ }^{67}$. Les mensonges appelés par leur nom, les Pseudéa petit-fils de Nuit, sont au contraire destinés aux mortels, comme l'ensemble de cette descendance ${ }^{68}$. Ce sont eux qu'Hermès introduit dans le corps de Pandora lors de sa fabrication, et ils constituent ainsi une composante de l'humanité actuelle, celle d'après Prométhée, que la première femme représente ${ }^{69}$. La relation étroite qu'Hésiode établit entre les "mensonges» et le "ventre" est particulièrement visible dans deux cas. Il s'agit d'abord d'une métaphore : les rois-juges, qui rendent des "sentences torses», non conformes aux faits et au droit, sont appelés par Hésiode $\delta \omega p o \varphi \alpha ́$ yot, "dévoreurs de présents", expression qui rend patent le caractère fallacieux de leurs dires par une image de voracité évoquant du même coup, bien entendu, leur vénalité $^{70}$. Il s'agit ensuite des $\psi \varepsilon v ́ \delta \varepsilon \alpha$ que les Muses revendiquent comme les leurs avant de proclamer leur connaissance des "vérités». Cette double compétence des Muses est énoncée immédiatement après l'adresse aux bergers, l'ensemble s'intégrant dans le récit de l'initiation d'Hésiode à la poésie. Rappelons ces paroles divines :

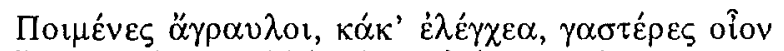

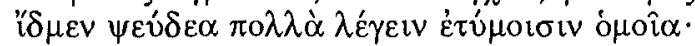

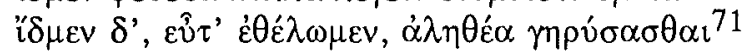

$J$ 'ai proposé ailleurs de comprendre ces «distorsions identiques à des paroles véridiques" non comme des "mensonges», au sens extrêmement péjoratif que le français donne à ce terme (car il y aurait bien de l'inconséquence de la part d'Hésiode à présenter les Muses comme menteuses au moment où elles le chargent de révéler l'être), mais comme des «fictions» renvoyant indirectement à la vérité, inaccessible à plus d'un mortel quand elle est formulée dans son plein éclat, qu'expriment les $\dot{\alpha} \lambda \eta \theta \dot{\varepsilon} \alpha$ du vers suivant ${ }^{72}$. Dans cette acception, les $\psi \varepsilon v ́ \delta \varepsilon \alpha$ sont synonymes de $\lambda \operatorname{co}_{\gamma} \varsigma_{\text {, }}$ «fiction, conte», et ce n'est pas par

67 Théog., 783-798.

68 Théog., 229.

69 Trav., 78.

70 Trau., 39, 221, 264.

71 Théog., 26-28. Le récit de l'initiation occupe les vers 22 à 34.

72 La parole chez Hésiode, thèse de doctorat d'État, 1990, Université de Paris XNanterre, à paraître. 
hasard si les Pseudéa et les Logoi voisinent dans la descendance de Nuit. Ce n'est pas par hasard non plus qu'Hésiode ne recourt à la fiction, nommée $\lambda$ óyos et $\alpha \hat{i} v o \varsigma$, que dans les Travaux ${ }^{73}$, où il s'adresse à un auditeur obtus, aussi peu disposé à recevoir la vérité qu'il l'est à travailler et à se conformer aux normes.

Il n'est donc nullement étonnant de retrouver chez Épiménide cette configuration sémantique, qui vient tout droit des représentations hésiodiques, synthétisées et poussées au noir. Dans son isolement, le vers n'en dit pas plus. Mais à ce silence, la légende épiménidienne vient heureusement remédier.

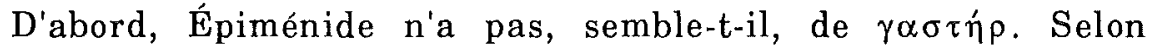
Plutarque, il ne mange pas ( $\alpha \dot{\delta} \varepsilon \imath \tau v o v)$ et, à en croire Diogène Laërce, ne produit pas d'excréments ${ }^{74}$. Adepte des simples, il consommait de la scille, de la mauve et de l'aspholède selon Théophraste et Plutarque ${ }^{75}$. Nourrisson des Nymphes dans son enfance, n'est-ce pas un aliment divin qu'il renfermait dans l'ongle d'un bœuf 76 ? N'étant pas un "ventre», encore moins serait-il une «bête». Son corps, d'où, selon la Souda, il s'éclipse à volonté, ne semble que l'enveloppe de son âme, qui, pendant son long sommeil, rencontre les dieux ${ }^{77}$. C'est pourquoi il est

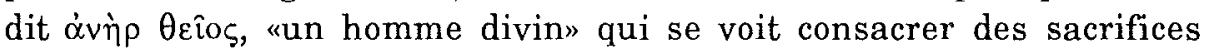

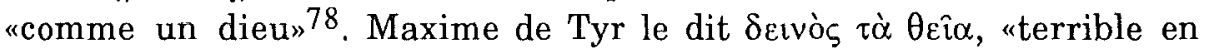
matière divine», et c'est à ce titre qu'il purifie Athènes ${ }^{79}$. On le dit "prophète» des dieux, dont il comprend la langue et qu'il rencontre en rêve. Parmi ces divinités, sont citées personnellement Dikè et les Alèthéiai, Justice et les Vérités ${ }^{80}$.

Sur plus d'un point, cette légende rappelle, elle aussi, des éléments hésiodiques, comme les anciens le notaient déjà. Plutarque affirme qu'Épiménide a trouvé chez Hésiode la recette de sa préparation de mauve et d'asphodèle ${ }^{81}$. Les vers auxquels il est fait allusion se situent dans les Travaux. S'en prenant aux rois «dévoreurs de présents», le

73 Trav., 106, 202.

74 PluT., Banquet, 157d; Diog. L., I, 114.

75 THEOPHR., Hist. pl., VII, 12, 1; PuuT., ibid.

76 DIOG. L., I, 114.

77 Souda, ibid. Des éléments analogues chez Diog. L.

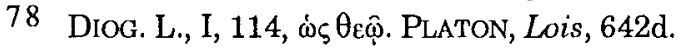

79 MAXIME DE TYR, c. 38, ap. fr. 1 Diels-Kranz.

80 Ibid. Sur Épiménide "maître de vérité», M. DETIENNE, Les maîtres de vérité dans la Grèce archaïque, Paris, 1967, notamment p. 129-131.

81 PLUT., Banquet, 157d. 
poète lance : «Nigauds ( plus que le tout, ni quel grand avantage se trouve dans la mauve et l'asphodèle» 82 . Considérés à juste titre comme peu clairs, ces vers opposent cependant nettement la frugalité à la voracité royale, et du même coup les droits jugements aux sentences fallacieuses. On en trouve confirmation dans le portrait du bon roi que figure Nérée : celui-ci est non seulement $\dot{\alpha} \psi \varepsilon v \delta \eta_{\zeta}$ et $\dot{\alpha} \lambda \eta \theta \eta \dot{\zeta}$, «sans mensonge et franc», mais il est ausi $\dddot{\eta} \pi \iota_{\zeta}$, "bienveillant», terme qui, par jeu de mots sinon par étymologie ${ }^{83}$, s'oppose à $v \eta ́ \pi ı$. Épiménide, familier de Justice et des Vérités, et en même temps nourri de mauve et d'asphodèle, "réalise», comme dit Platon 84 , le type de sage auquel rêve Hésiode. Ce dernier d'ailleurs comprenait les Muses et pouvait reproduire leurs chants ou les traduire aux hommes ordinaires, de même qu'Épiménide comprend les paroles des dieux.

Il y a cependant une différence radicale entre les deux types de rapport aux dieux qu'entretiennent les deux sages. Hésiode a reçu la

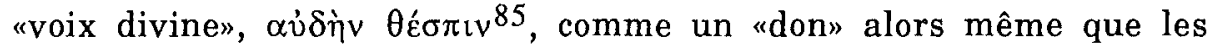
Muses viennent de l'appeler, au même titre que les autres bergers, un «ventre». Il s'agit bien sûr d'une capacité d'origine divine qui hisse le poète au-dessus du commun; mais c'est là une compétence supplémentaire conférée une fois pour toutes et qui ne remet pas fondamentalement en cause la nature mortelle du poète. Toutes les leçons de la Théogonie et des Travaux invitent à une juste appréciation de la situation de l'homme dans le monde, et on ne voit nulle part qu'Hésiode échapperait au sort commun, quelque informé qu'il soit de la vérité divine. En outre, la rencontre avec les Muses a lieu à l'Hélicon, où se trouve, certes, un autel, mais où paissent aussi les troupeaux d'Hésiode. Il ne s'agit pas d'un lieu proprement divin. D'Épiménide en revanche, seul le corps demeure dans la grotte crétoise. Sa rencontre avec les dieux a lieu chez eux, à chaque fois qu'il le désire et à son initiative, semble-t-il. Il y a, en lui comme chez Hésiode, quelque chose de divin, mais cette chose est mobile par rapport à la partie mortelle du sage, dont elle diffère et qu'elle ne peut entraîner à sa suite dans ses consultations divines.

82 Trav., 40-41.

83 Voir Chantraine, Dictionnaire, s.v. $\ddot{n} \pi \mathrm{to}$, mais aussi J.P. Vernant, Sur le vieux de la mer chez Hésiode, in REG, 76 (1963), p. XVII-XvIII. Le passage sur Nérée est en Théog., 233-236, où on note le doublet $\ddot{\eta} \pi$ เо...

84 Platon, Lois, 677d.

85 Théog., 31-32. 
Entre Hésiode et Épiménide, l'homme, à tout le moins le prophète, a acquis une âme, notion que le poète béotien n'avait à aucun degrée ${ }^{86}$.

Ainsi Épiménide "réalise» Hésiode là-même où il s'en écarte. Sa pilule de simples et ses réprimandes aux crétois rejettent le corps du côté du bestial d'une manière plus radicale que chez Hésiode. Un tel infléchissement n'est possible que grâce à l'existence "effective» de la recette purement «verbale» des Travaux 87 . Car la composition de mauve et d'asphodèle, si elle permet de survivre, suppose qu'un homme, du moins un sage, ne se réduit pas à son corps. On voit comment la notion d'âme, principe distinct de l'enveloppe corporelle qui lui est comme une maison, se constitue en prenant étroitement appui sur des conceptions hésiodiques dont cependant elle s'écarte de manière significative. Ce que la confrontation entre Hésiode et Épiménide permet de saisir, c'est le passage d'une conception moniste de l'homme, qui était celle de la pensée mythique, au dualisme somato-psychique cher à la philosophie. Ainsi, quelles que soient sa chronologie, ses origines, l'effectivité de ses actes ou l'authenticité de ses propos, la date d'Épiménide est celle de ce passage : il est l'emblème de l'apparition en Grèce du dualisme somato-psychique.

31 , rue des Hautes Bornes

Marie-Christine LECLERC

F - 94310 ORLY

86 Пl y a un seul emploi de $\psi v \chi \eta^{\prime}$ chez Hesiode (Trav., 686), dans le sens de uce qui est toute la vie».

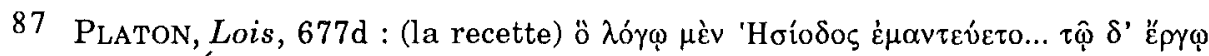



